\title{
EDITORIAL
}

\section{Staging in bipolar disorder: one step closer to precision psychiatry}

\author{
Brisa S. Fernandes, ${ }^{1,2}$ Michael Berk ${ }^{1,3,4}$ \\ ${ }^{1}$ IMPACT Strategic Research Centre and Barwon Health, School of Medicine, Deakin University, Geelong, VIC, Australia. ${ }^{2}$ Laboratório das \\ Proteínas Ligantes do Cálcio no Sistema Nervoso Central, Departamento de Bioquímica, Universidade Federal do Rio Grande do Sul \\ (UFRGS), Porto Alegre, RS, Brazil. ${ }^{3}$ Department of Psychiatry, University of Melbourne, Parkville, Melbourne, VIC, Australia. ${ }^{4}$ Florey Institute \\ for Neuroscience and Mental Health, Department of Psychiatry and Orygen, The National Centre of Excellence in Youth Mental Health, \\ University of Melbourne, Parkville, Melbourne, VIC, Australia.
}

The need to bring the new paradigm of precision medicine to psychiatry - precision psychiatry - has been acknowledged. ${ }^{1}$ In this sense, the article by Brietzke et al. ${ }^{2}$ covers one of the most important contemporary topics in bipolar disorder, which is the recognition of the specificity of its early stages. As is highlighted by the authors, proper recognition of the early stages of the disease is indispensable in order to implement strategies capable of avoiding, or at least delaying, the progression to later stages related to neuroprogression, which are associated with marked disability, as well as for development of preventive initiatives.

At the core of the identification of an early stage of disease is the concept of staging, which implies that bipolar disorder has a progressive course, at least in a subgroup of persons. This has led to an increased interest in research considering stage models in the last few years. The concept itself is not new: Kraepelin, despite not using the term, recognized the occurrence of disease stages, as can be inferred by his observation that psychiatric disorders in general could progress over time and episodes in bipolar disorder in particular would recur more rapidly.

The proposed staging model is adapted from the fourstage model used in general medicine. When applied to bipolar disorder, as pointed out by the authors, the disorder starts during an "at-risk period" and progresses to the first mood episode and follows to a "late stage." This would be a consequence of the phenomenon of neuroprogression, which is considered to be caused, at least in part, by an increase in inflammation and immune mediated stress, leading to a decrease in neurotrophic factors and consequent brain rewiring. ${ }^{3,4}$

It is thought that bipolar disorder indeed progresses, but one point that needs further clarifying is whether this is a universal phenomenon. The majority of research until now was conducted in specialized centers, where the sample studied is usually biased towards including persons with a more severe and chronic form of the disease. Another limitation is that, in virtually all studies that assessed cognition, neuroimaging, and biomarkers - all domains that are considered in the core of the staging model - the assessment of these markers was not done in participants

Correspondence: Brisa S. Fernandes, Barwon Health, School of Medicine, Deakin University, Geelong, VIC, Australia.

E-mail: brisasf@gmail.com categorized using the stages considered to compose the model, but instead often used length of illness or functioning as proxies for staging. This has two major consequences: first, these studies cannot properly assess and therefore validate the proposed stages that compose the model; second, it assumes not only that neuroprogression occurs at least in the great majority of persons with the disease, but also that the course of neuroprogression follows a relatively linear pattern. However, further studies are needed before the veracity of either of these assumptions is proved.

It is plausible that the natural course of bipolar disorder is not linear, and this is actually acknowledged in the staging model itself when it proposes that appropriate treatment in the early and intermediate stages might ameliorate or even reverse damage. ${ }^{3,4}$ Regarding linearity and analysis of blood-based biomarkers, results from meta-analyses have shown that levels of brain-derived neurotrophic factor, ${ }^{5}$ the most abundant neurotrophin, and of C-reactive protein, ${ }^{6}$ a validated biomarker of systemic inflammation, are not associated with length of illness, which might suggest that the progress of the disease is not inexorable or that alterations in (at least these two) blood-based biomarkers are not perfectly aligned with length of illness. These are considerations that need to be clarified. At any rate, it appears that simply employing length of illness as a proxy for staging has limitations and that studies classifying participants in the proposed stages of the model are in order.

Finally, it is important to keep in mind that the staging model would greatly benefit from discovery of biomarkers that could lead to development of tools capable of guiding clinicians in their assessment and, consequently, to the most appropriate intervention. To enable such development, these biomarkers would have to prove their value beyond simply informing about pathophysiology: they must also have proven clinical validity, which is, broadly speaking, demonstrated by high accuracy, sensitivity, and specificity; however, to date, very few studies have assessed the validity of these parameters for staging. ${ }^{7}$ It is also important to keep in mind that a biomarker capable of accurately identifying stages in the model is not necessarily useful or valid for diagnosis of bipolar disorder: these biomarkers will most likely be shared across other disorders, most remarkably schizophrenia and major depression, 
and therefore unspecific and without diagnostic value for bipolar disorder. At present, this is still merely an aspiration, as biomarkers with sufficient sensitivity and specificity remain elusive.

Future studies should also benefit from the advanced analytical methods employed by machine-learning techniques capable of making predictions at an individual level, as is currently advocated in precision psychiatry. ${ }^{1}$ Only when these issues have been clarified will the concept of staging translate into improved care and, hopefully, improved outcomes. It remains a truism across medicine that early treatment is generally superior to late treatment, and the early intervention movement allied to the staging concept remains a promising approach to potentially devastating disorders.

\section{Disclosure}

The authors report no conflicts of interest.

\section{References}

1 Fernandes BS, Williams LM, Steiner J, Leboyer M, Carvalho AF, Berk M. The new field of 'precision psychiatry'. BMC Med. 2017;15:80.

2 Brietzke E, Rosa AR, Pedrini M, Noto MN, Kapczinski F, Scott J. Challenges and developments in research of the early stages of bipolar disorder. Rev Bras Psiquiatr. 2016;38:329-37.

3 Berk M, Berk L, Dodd S, Cotton S, Macneil C, Daglas R, et al. Stage managing bipolar disorder. Bipolar Disord. 2014;16:471-7.

4 McGorry P, Keshavan M, Goldstone S, Amminger P, Allott K, Berk M, et al. Biomarkers and clinical staging in psychiatry. World Psychiatry. 2014;13:211-23.

5 Fernandes BS, Molendijk ML, Kohler CA, Soares JC, Leite CM, Machado-Vieira $R$, et al. Peripheral brain-derived neurotrophic factor (BDNF) as a biomarker in bipolar disorder: a meta-analysis of 52 studies. BMC Med. 2015;13:289.

6 Fernandes BS, Steiner J, Molendijk ML, Dodd S, Nardin P, Goncalves $\mathrm{CA}$, et al. C-reactive protein concentrations across the mood spectrum in bipolar disorder: a systematic review and meta-analysis. Lancet Psychiatry. 2016;3:1147-56.

7 Kapczinski F, Fernandes BS, Kauer-Sant'Anna M, Gama CS, Yatham LN, Berk M. The concept of staging in bipolar disorder: the role of BDNF and TNF-alpha as biomarkers. Acta Neuropsychiatr. 2009;21:272-4. 